\title{
A hyposensitive anticancer drug induces higher surface expression and release of heat shock proteins in a human hepatocellular carcinoma cell line
}

\author{
MEI YANG ${ }^{1-3}, \mathrm{ZHE} \mathrm{XU}^{4}$, QI WANG ${ }^{1}, \mathrm{AN}-Q I N ~ Z H A N G^{1}$ and JUN MIN ${ }^{3}$ \\ ${ }^{1}$ Breast Disease Center, Guangdong Women and Children Hospital of Guangzhou Medical University, Guangzhou, \\ Guangdong 510010; ${ }^{2}$ Department of General Surgery, General Hospital of Guangzhou Military Command of PLA, \\ Guangzhou, Guangdong 510010; ${ }^{3}$ Department of Hepatobiliary Surgery, Sun Yat-Sen Memorial Hospital, \\ Sun Yat-Sen University, Guangzhou, Guangdong 510120; ${ }^{4}$ Department of Ophthalmology, \\ General Hospital of Guangzhou Military Command of PLA, Guangzhou, Guangdong 510010, P.R. China
}

Received June 30, 2014; Accepted March 24, 2015

DOI: $10.3892 / \mathrm{mmr} .2015 .3727$

\begin{abstract}
Heat shock proteins (HSPs) respond to multiple stresses and have been implicated as essential immune chaperones that regulate innate and adaptive immunity. The exposure of HSPs containing tumour peptide complex to immune surveillance elements may elicit a specific anti-tumour response. The present study examined the potential of anticancer drugs to induce apoptosis of HepG2 cells and elicit the expression of HSP proteins, including HSP70 and gp96, on the membrane or their release to the extracellular environment, leading to HSP exposure. In the present study, etoposide and carboplatin were classified by an adenosine triphosphate assay as representatives of hypersensitive and hyposensitive anticancer drugs, respectively. Flow cytometry, immunofluorescence, ELIZA and reverse transcription quantitative polymerase chain reaction were all used to detect changes in the HSPs. The results demonstrated that etoposide and carboplatin induced apoptosis of HepG2 cells. In addition, following treatment with etoposide or carboplatin, HSP70/gp96 expression increased, demonstrating a 'transfer expression' pattern: The cytosol expression decreased while the surface expression increased. These alterations progressed steadily with notable alterations following treatment with etoposide for $24 \mathrm{~h}$ or carboplatin for $72 \mathrm{~h}$. Additionally, at the end of treatment, release of
\end{abstract}

Correspondence to: Professor Jun Min, Department of Hepatobiliary Surgery, Sun Yat-Sen Memorial Hospital, Sun Yat-Sen University, 107 Janjiang West Road, Guangzhou, Guangdong 510120, P.R. China

E-mail:drminj@qq.com

Dr Mei Yang, Breast Disease Center, Guangdong Women and Children Hospital of Guangzhou Medical University, 13 Guangyuan West Road, Guangzhou, Guangdong 510010, P.R. China

E-mail: bayberry513@yahoo.com

Key words: hepatocellular carcinoma, chemotherapy, heat shock proteins
HSP70/gp96 to the extracellular environment increased. Notably, following treatment with the hyposensitive anticancer drug carboplatin for $72 \mathrm{~h}$, the surface expression of gp96 in HepG2 cells was significantly increased. These results suggest that when combined with cancer cell apoptosis, anticancer drugs induce the membrane expression and release of HSP70/ gp96 in hepatocellular carcinoma (HCC) cells, which may represent a crucial event in the immune anti-tumour response. Notably, treatment with the hyposensitive anticancer drug for a longer time period resulted in greater surface expression and release of gp96, which suggests a potential use for hyposensitive anticancer drugs in HSP-based dendritic cell vaccine preparation and chemoimmunotherapy for HCC patients.

\section{Introduction}

Hepatocellular carcinoma (HCC) is a type of tumour with increasing incidence and a high mortality rate worldwide, ranking as the second most prevalent cancer in China (1-4). However, since it remains clinically asymptomatic until the late stages, therapeutic options are limited. Even at the early stages, treatments, including partial liver resection, chemotherapy, radiotherapy and molecular targeted therapy have demonstrated only a modest clinical benefit (5). Since HCC has been demonstrated to be immunogenic (6), immunotherapy, which aims at inducing the immune system to eradicate malignant, transformed cells, is considered to be a potential approach for the treatment HCC (7). However, in clinical practice, chemotherapy $(8,9)$ or immunotherapy $(10)$ alone cannot achieve satisfactory therapeutic efficacy. Thus, the use of chemoimmunotherapy has been proposed. The putative theory is that traditional chemotherapy can lower the tumour burden and promote antigen presentation, which elicits an immunoresponse. A therapeutic chemodrug strategy using GOLF (gemcitabine, oxaliplatin, leucovorin and 5-fluorouracil), followed by subcutaneous granulocyte macrophage colony-stimulating factor and interleukin-2 to treat metastatic colorectal carcinoma patients resulted in a good therapeutic response and disease control rate and 
effectively delayed disease progression (11). In vitro, it was found that GOLF therapy elicited an antigen-specific cytotoxic T lymphocyte (CTL) immune response mediated by dendritic cells (DCs) (12). This immune reaction may be due to the presentation of a 'risk signal' induced by chemodrugs (12). It is well established that chemotherapy causes tumour cell apoptosis (13); however, apoptotic cells stimulate variable outcomes (i.e., immunotolerance or immunoreactions) under different conditions. A previous study demonstrated that apoptotic cells induced by radioactive rays or retrogradation suppress immunoreactions as the immune system interprets this apoptotic phenomenon as normal (14). By contrast, apoptotic cells induced by infection, heat shock or certain chemodrugs could activate antigen-presenting cells and then elicit an immune reaction; this apoptosis was considered to be a 'danger' and thus abnormal (15). Therefore, tumour cells were induced to undergo apoptosis and to express heat shock proteins (HSPs), well-known protein chaperone molecules, by gene transfection to elicit a 'danger signal' and enhance tumour immunogenicity. Notably, when combined with 'danger signal' HSPs, apoptotic tumour cells elicited an immune response and subsequently destroyed residual tumour cells (16).

HSPs, including HSP70, gp96, HSP27 and HSP60, are intracellular molecular chaperones of nascent proteins and function during protein synthesis, folding, assembly, transport and stabilization (8). HSPs are synthesised under different types of stress conditions, including cell growth and differentiation, infection, inflammation, malignancy, heat shock and oxygen radicals (17). An immune response is elicited by HSPs isolated from cancer cells, not from normal cells, due to the tumour antigen peptide associating with HSPs. In this response, HSPs act as a 'danger signal' to attract and activate DCs (18). Those HSPs eliciting tumour immunogenicity are marked not by the constitutive but by the inducible HSPs (19), which may be exposed under the immune surveillance elements. Thus, the exposure of HSPs represents an important event in the anti-tumour response (17).

A comparison of HCC and normal samples demonstrated HSP deregulation in tumour cells (20). A comparison of HCC and normal samples demonstrated HSP deregulation in tumour cells (20). HSP70 and gp96 are stress sensitive members of the HSP family and have been the focus of numerous studies $(21,22)$. Using flow cytometry, confocol microscopy, ELISA and reverse transcription quantitative polymerase chain reaction, the present study examined the potential of anticancer drugs to induce apoptosis of HepG2 cells and determine the protein expression levels of HSPs, including HSP70 and gp96, on the membrane or their release into the extracellular environment, leading to HSP exposure.

\section{Materials and methods}

Cell culture and reagents. The human HCC cell line HepG2 used in the present study was purchased from the American Type Culture Collection (Manassas, VA, USA) and routinely maintained in complete Dulbecco's modified Eagle's culture medium (25 mM D-glucose, $4 \mathrm{mM}$ L-glutamine and $1 \mathrm{mM}$ sodium pyruvate; Gibco-BRL, Carlsbad, CA, USA) supplemented with $10 \%$ foetal bovine serum (Gibco-BRL) at $37^{\circ} \mathrm{C}$ in $5 \% \mathrm{CO}_{2}$.
Growth inhibition assay. An adenosine triphosphate-tumour chemosensitivity assay (ATP-TCA; Biothema AB, Handen, Sweden) was used to determine the growth inhibitory effects of anticancer drugs on HepG2 cells. All anticancer drugs, including paclitaxel [100\% test drug concentration (TDC) $13.6 \mu \mathrm{g} / \mathrm{ml}$; Bristol-Myers Squibb Co., New York, NY, USA], etoposide (100\% TDC $48 \mu \mathrm{g} / \mathrm{ml}$; Jiangsu Hengrui Medicine Co., Nanjing, China), pharmorubicin (100\% TDC $0.5 \mu \mathrm{g} / \mathrm{ml}$; Pfizer, Inc., New York, NY, USA), carboplatin (100\% TDC $15.8 \mu \mathrm{g} / \mathrm{ml}$; Bristol-Myers Squibb Co.), irinotecan hydrochloride (100\% TDC $14 \mu \mathrm{g} / \mathrm{ml}$; Pfizer, Inc.) and mitomycin (100\% TDC $0.23 \mu \mathrm{g} / \mathrm{ml}$; Zhejiang Hisun Pharmaceutical Co., Taizhou, China) were prepared according to the manufacturer's instructions of the ATP-TCA kit. In total, $3 \times 10^{3}$ cancer cells growing in a 96-well plate were incubated with different anticancer drugs at different concentrations [6.25, 12.5, 25, 50, 100 and 200\% test drug concentration (TDC)] in complete growth medium at $37^{\circ} \mathrm{C}$ and $5 \% \mathrm{CO}_{2}$. The cells were collected for the ATP-TCA assay $72 \mathrm{~h}$ later according to the manufacturer's instructions. Specifically, $100 \mu \mathrm{l}$ ATP extraction solution was added to the cells, mixed and incubated for 20-30 min at room temperature. Following that, $500 \mu \mathrm{l}$ of the mixture was added to the detection plate and mixed with $50 \mu \mathrm{l}$ fluorescence-luciferase. It was then analysed using a Luminescence analyser $\left(\right.$ Synergy ${ }^{\mathrm{TM}}$ MX; BioTek, Winooski, VT, USA). Data were analysed by Microsoft Excel 2010 software and hyper- and hyposensitive drugs of HepG2 cells were profiled.

Anticancer drug treatment on HepG2 cells. The hypersensitive drug etoposide and the hyposensitive drug carboplatin were selected for the experiments. HepG2 cells were cultured in a 6-well plate at $37^{\circ} \mathrm{C}$ and $5 \% \mathrm{CO}_{2}$. Etoposide or carboplatin with $100 \%$ TDC was added and co-cultured for the indicated time intervals: $1,2,4,6,12,18,24,48$ or $72 \mathrm{~h}$. The etoposide-treated group was not assessed for 48 or $72 \mathrm{~h}$ as it caused marked apoptosis after $24 \mathrm{~h}$ of treatment. At each time interval, the cells were harvested for flow cytometry or immunostaining analysis.

Flow cytometry. Following treatment with anticancer drugs, cancer cells were collected for intracellular and surface immunolabelling of HSP70/gp96. Briefly, for intracellular labelling, cells were fixed and permeabilised using $70 \%$ ethanol at $4{ }^{\circ} \mathrm{C}$ overnight and then incubated with each of the following antibodies: Phycoerythrin-conjugated mouse anti-HSP70 polyclonal antibody (1:100; cat. no. sc-1060; Santa Cruz Biotechnology, Inc., Santa Cruz, CA, USA); rabbit anti-human GRP94 (gp96; 1:100; cat. no. AHP848; AbD Serotec, Raleigh, NC, USA) followed by the secondary antibody [goat anti rabbit-fluorescein isothiocyanate (FITC); 1:200; cat. no. 172-1506; Kirkegaard \& Perry Laboratories Inc., Gaithersburg, MD, USA] for $20 \mathrm{~min}$ at room temperature and protected from light. For surface HSP70 or gp96 staining, tumour cells were dissociated into a single cell suspension and incubated under the same conditions as described above without fixation and permeabilization. All labelled cells were washed and measured immediately using a FACS Calibur flow cytometer (Beckman-Coulter, Inc., Miami FL, USA). More than $1 \times 10^{4}$ cells were analysed in each analysis and the mean fluorescence intensity (MFI) was used for the assessment 
of HSP70 and gp96 expression. Controls were routinely performed in all cases.

Immunofluorescence. Tumour cells were incubated with hypersensitive or hyposensitive drugs in a 6-well plate with a coverslip for different time intervals: Etopiside for $24 \mathrm{~h}$ and carboplatin for $72 \mathrm{~h}$. Coverslips with cells were collected for indirect immunofluorescence staining. Cells were fixed and permeabilised using $70 \%$ ethanol at $4^{\circ} \mathrm{C}$ overnight and blocked by normal goat serum confining liquid for $30 \mathrm{~min}$ at room temperature. Subsequently, the cells were incubated with the following specific antibodies for $1 \mathrm{~h}$ at room temperature: Mouse anti-HSP70 (1:100; cat. no. BM0368; Wuhan Boster Biological Technology, Ltd., Wuhan, China) and rabbit anti-GRP94 (gp96) monoclonal antibody (1:100; cat.no. AHP848; ABD Serotec, Inc.). Secondary antibodies used for the visualization of HSP70 or gp96 were FITC-conjugated goat anti-mouse IgG (Wuhan Boster Biological Technology, Ltd.) and Cy3-conjugated sheep anti-rabbit IgG (Sigma-Aldrich, St. Louis, MO, USA). Hoechst was used for nuclear staining. During incubation with secondary antibodies and Hoechst, the cells were protected from light. Control staining was performed in all cases. Following staining, the cells were observed and images were captured under a fluorescent confocal microscope $(\mathrm{LS}<510$ META; Carl Zeiss, Jena, Germany).

Apoptosis. An annexin $\mathrm{V}$ detection kit used to determine apoptosis was provided by BioVision, Inc. (Milpitas, CA, USA; cat. no. K102-25). Tumour cells treated with drugs were dissociated into single cell suspensions in $200 \mu \mathrm{l}$ binding buffer. Subsequently, $10 \mu 1$ FITC-conjugated annexin V and $5 \mu \mathrm{l}$ propidium iodide were added, and then the cells were incubated for $30 \mathrm{~min}$ at room temperature while protected from light. Next, $300 \mu \mathrm{l}$ binding buffer was added and the cells were analysed using a FACS Calibur flow cytometer (Beckman-Coulter, Inc.).

ELISA. HSP70 (Surveyor ${ }^{\mathrm{TM}}$ IC; R\&D Systems, Minneapolis, MN, USA; cat. no. SUV1663) and gp96 (MyBioSource, San Diego, CA, USA; cat. no. MBS705033) ELISA kits were used and the quantity of released HSP70/gp96 from HepG2 cells treated with chemodrugs was analysed according to the manufacturer's instructions. Briefly, culture supernatants in which HepG 2 cells were cultured with etoposide for $24 \mathrm{~h}$ or carboplatin for 24 or $72 \mathrm{~h}$ were collected and $100 \mu \mathrm{l}$ of them were added to the microtitre that was pre-coated with anti-HSP70 or gp96 antibody. The cells were then incubated for $2 \mathrm{~h}$ at room temperature and washed three times with PBS. Subsequently, $100 \mu 1$ HSP70 or gp96 detection antibody was added and incubated for $2 \mathrm{~h}$ at room temperature. The HSP70 or gp96 antibody were washed off and streptavidin horseradish peroxidase (HRP; HSP70) or HRP avidin (gp96) was added and incubated at room temperature for an additional $20 \mathrm{~min}$.Following that, $100 \mu \mathrm{l}$ substrates were added and incubated for $20 \mathrm{~min}$. Finally, $50 \mu \mathrm{l}$ stop solution was added and the optical density was determined at $450 \mathrm{~nm}$ using a Luminescence analyzer. The HSP70 or gp96 standard was used to make a standard curve by proportional dilution and the formula was produced for the concentration and optical density. Subsequently, the HSP70 or gp96 concentration in each sample was calculated.
Reverse transcription quantitative polymerase chain reaction (RT-qPCR). HepG2 cells treated with etoposide for $24 \mathrm{~h}$ and carboplatin for 24 or $72 \mathrm{~h}$ were harvested. Total RNAs were extracted and reverse transcribed into cDNA using Superscript II reverse transcriptase (Invitrogen Life Technologies, Carlsbad, CA, USA). RT-qPCR was performed using the Lightcycler-Faststart DNA master SYBR green I PCR kit (Roche Diagnostics, Madison, WI, USA) in a Roche Lightcycler 1.2 Real Time PCR System (Roche Diagnostics) according to the manufacturer's instructions: Initiation with a 10-min denaturation at $95^{\circ} \mathrm{C}$, followed by 40 cycles of amplification with $15 \mathrm{sec}$ of denaturation at $95^{\circ} \mathrm{C}, 5 \mathrm{sec}$ of annealing at $50-60^{\circ} \mathrm{C}$, $15 \mathrm{sec}$ of extension at $72^{\circ} \mathrm{C}$, and then the plate was read for fluorescence data collection at $76^{\circ} \mathrm{C}$. The primer sequences were as follows: HSP70, forward 5' TGGTGGTTCTACTCG TATCCC-3' and reverse 5'-TGACATCCAAGAGCAGCA AAT-3'; Gp96,forward5'-GCTTCGGTCAGGGTATCTTTT-3' and reverse 5'-CACCTTTGCATCAGGGTCAAT-3'; GAPDH, forward 5'-TGTTGCCATCAATGACCCCTT-3' and reverse 5'-CTCCACGACGTACTCAGCG-3'. The comparative threshold cycle (CT) method was used for the calculation of amplification fold. The expression level of each gene was normalised by dividing by the expression level of the GAPDH gene transcript.

Statistical analysis. All data are expressed as the mean \pm standard deviation. Statistical analyses were performed using paired Student's t-test with Microsoft Excel 2010 (Microsoft, Albuquerque, NM, USA). $\mathrm{P}<0.05$ was considered to indicate a statistically significant difference.

\section{Results}

Chemosensitivity of HepG2 cells to anticancer drugs and apoptosis caused by hyper-or hyposensitive anticancer drugs. To determine the chemosensitivity of HepG2 cells to anticancer drugs, the present study selected six commonly used drugs to treat HepG2 cells and then used those cells to conduct ATP-TCA assays. For each single anticancer drug, the chemosensitivity was categorised as sensitive (100\% TDC>90\% and $50 \% \mathrm{TDC}<70 \%)$ or resistant $(100 \% \mathrm{TDC}<70 \%$ and $50 \%$ TDC $>50 \%$ ). Dose-response curves for HepG2 cells following a continuous $72 \mathrm{~h}$ exposure to anticancer drugs at various concentrations using the ATP-TCA assay are depicted. The results demonstrated that etoposide (VP-16), taxol (TAX) and pharmorubicin had high inhibitory rates and were thus classified as sensitive drugs. By contrast, mitomycin, irinotecan and carboplatin had low inhibitory rates (Fig. 1A and B) and were classified as resistant drugs. According to the results, the hypersensitive drug etoposide (VP-16), a cell cycle-specific anti-tumour drug that targets the $\mathrm{S}$ phase or $\mathrm{G} 2$ phase and the hyposensitive drug carboplatin, a cell cycle-non-specific drug, which inhibits DNA duplication and transcription, were selected for subsequent experiments. Subsequently, HepG2 cells were treated with hypersensitive (etoposide) or hyposensitive (carboplatin) drugs for the indicated time intervals and cell apoptosis levels were determined using an annexin $\mathrm{V}$ apoptosis detection kit. Apoptosis of drug-treated HepG2 cells slowly increased with time. Etoposide caused marked apoptosis in a relatively short time period, with 
A

B
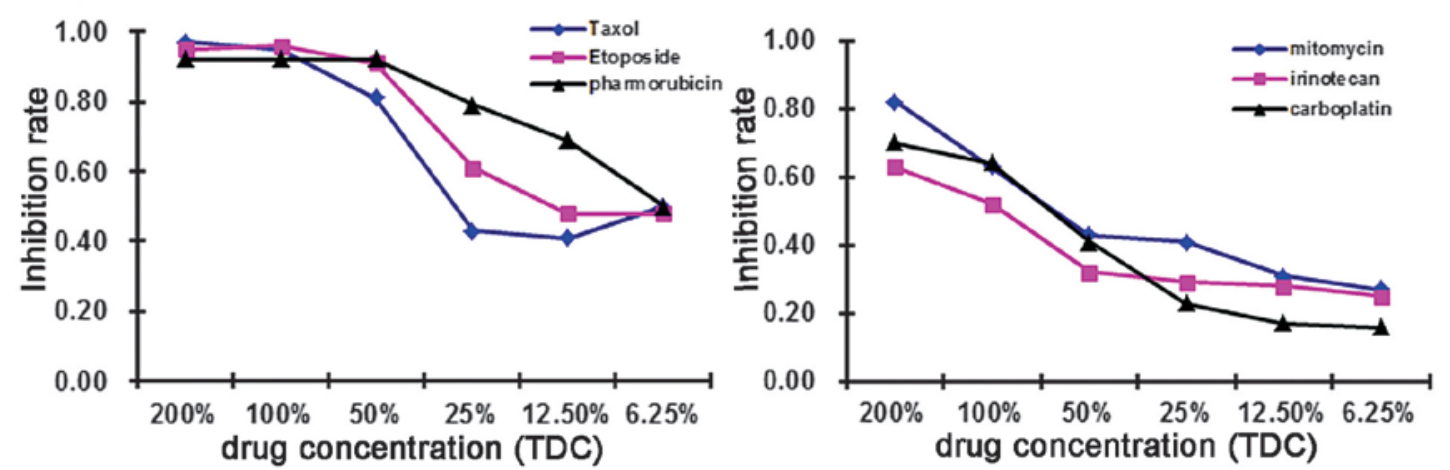

C

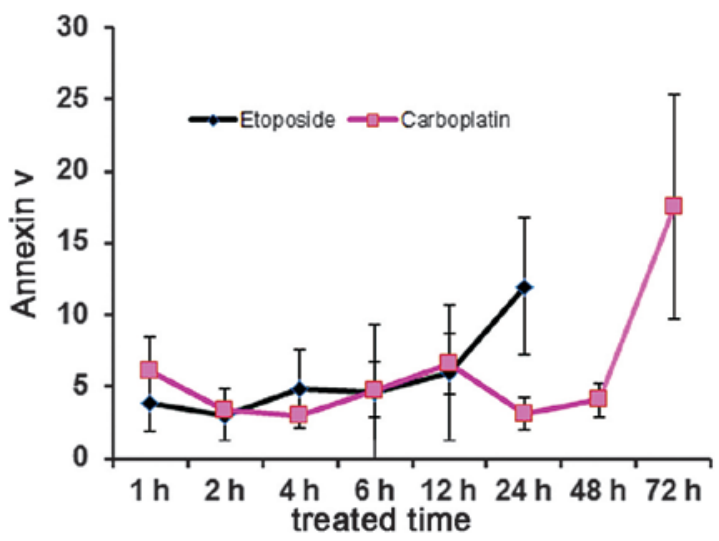

Figure 1. Chemosensitivity of HepG2 cells to anticancer drugs and apoptosis caused by hyper- or hyposensitive anticancer drugs. Dose-response curves for HepG2 cells following continuous $72 \mathrm{~h}$ exposure to anticancer drugs at various concentrations using the adenosine triphosphate-tumour chemosensitivity assay are depicted. HepG2 cells demonstrated significantly higher sensitivity to (A) etoposide (VP-16), taxol (TAX) and pharmorubicin compared with resistance to (B) mitomycin, irinotecan and carboplatin. The data shown are representative of four independent experiments for each drug. (C) HepG2 cells were treated with hypersensitive (etoposide) or hyposensitive (carboplatin) drugs for the indicated time intervals and cancer cell apoptosis was determined using an annexin $\mathrm{V}$ apoptosis detection kit.

the maximal annexin $\mathrm{V}$ positive rate achieved after $24 \mathrm{~h}$. Carboplatin-treated cells took longer $(72 \mathrm{~h})$ to arrive at the maximal annexin $\mathrm{V}$ positive rate (Fig. 1C).

Alterations in the mRNA level and secretion of HSP70/gp96 in etoposide- or carboplatin-treated HepG2 cells. HepG2 cells were treated with etoposide for $24 \mathrm{~h}$ or carboplatin for $72 \mathrm{~h}$. The cells were lysed and the mRNA levels of HSP70/gp96 were determined by RT-qPCR (Fig. 2A and B). In addition, cell culture supernatants were collected and the quantity of secretory HSP70 or gp96 was quantified by ELISA (Fig. 2C and D). The data indicated that although etoposide and carboplatin increased the mRNA level of HSP70 and gp96 in HepG2 cells, only carboplatin-treated HepG2 cells produced increased secretory HSP70 and gp96.

Gradual alterations in cytoplasmic and surface HSP70/gp96 expression in etoposide-or carboplatin-treated HepG2 cells. HepG2 cells were treated with etoposide or carboplatin for the indicated time intervals: $0,1,2,4,6,12,18,24,48$ and $72 \mathrm{~h}$. The cytoplasmic and surface HSP70 (Fig. 3A) and gp96 (Fig. 3B) expression levels were determined by flow cytometry and the parameter MFI was used to quantify the alterations. As shown in Fig. 3, prior to treatment with chemodrugs, HepG2 cells exhibited strong intracellular (cytoplasmic) HSP70/gp96 expression but weak surface expression. However, following treatment with chemodrugs, cytoplasmic HSP70/gp96 expression decreased and cancer cells exhibited upregulated surface HSP70/gp96 expression. Marked alterations were observed following $24 \mathrm{~h}$ treatment with etoposide and $72 \mathrm{~h}$ treatment with carboplatin. Following treatment with carboplatin for $72 \mathrm{~h}$, the surface expression of gp96 in HepG2 cells was significantly increased.

Cytoplasmic and surface expression alterations in HSP70/gp96 in drug-treated HepG2 cells. HepG2 cells were treated with etoposide for $24 \mathrm{~h}$ or carboplatin for $72 \mathrm{~h}$ and immunofluorescence staining was used to demonstrate HSP70/gp96 expression. Consistent with flow cytometric analysis, the confocal images demonstrated a decrease in cytoplasmic HSP70/gp96 expression (Fig. 4A) and a clear increase in the surface expression of HSP70/gp96 (Fig. 4B). However, the increase in the surface expression of HSP70 was not significant.

\section{Discussion}

Almost all cells contain HSPs, which are present in a variety of intracellular locations, including the cytosol, endoplasmic reticulum, nuclei and mitochondria. HSPs function in normal cells as molecular chaperones to assist protein folding, unfolding, degradation and assembly (8). When cells become 

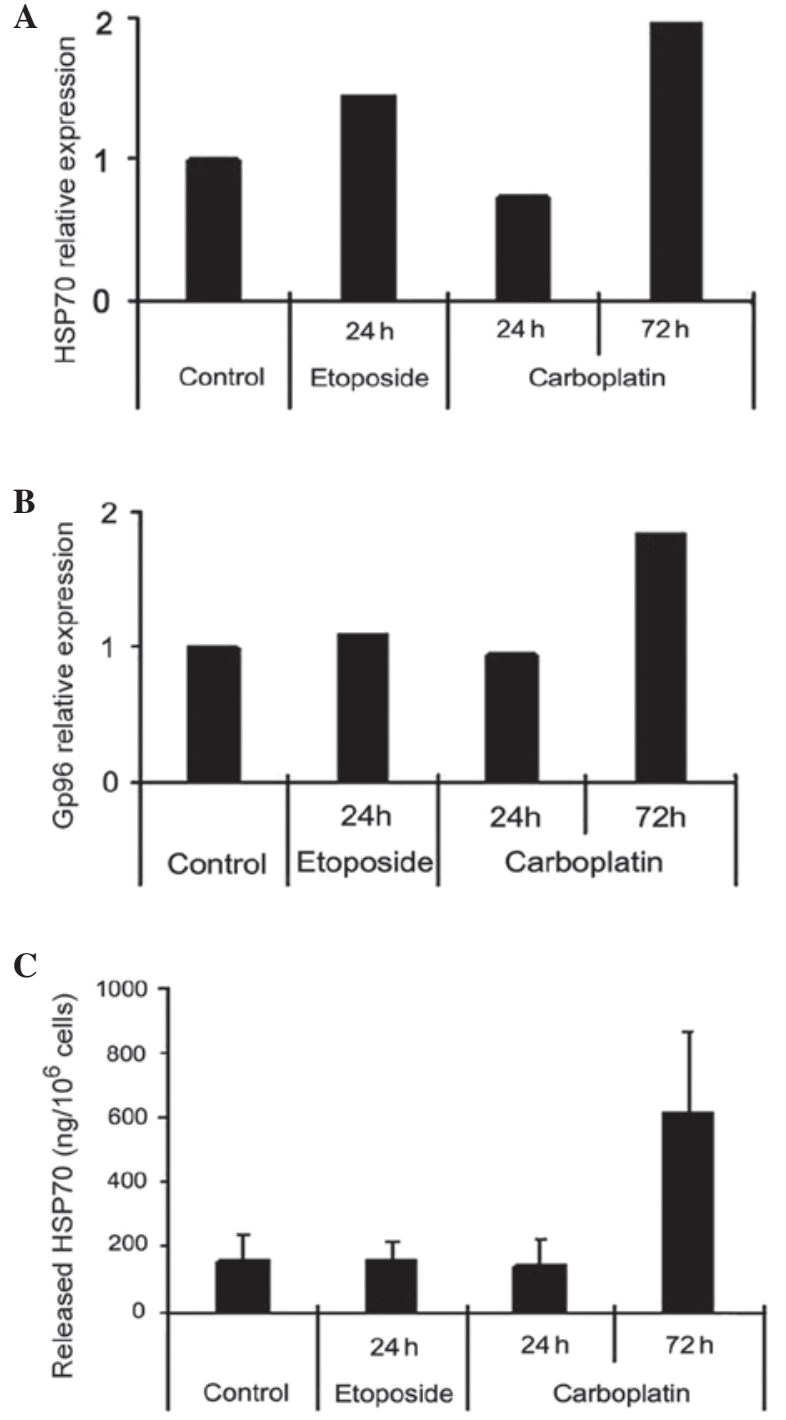

D

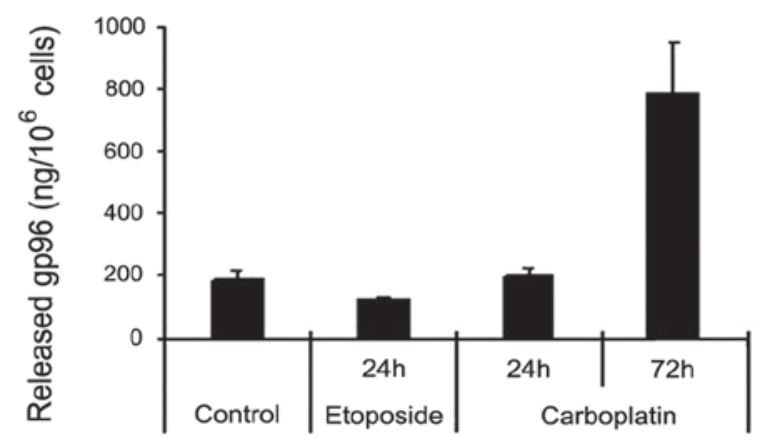

Figure 2. Alterations in the mRNA levels and quantity of secretory HSP70 and gp96 in etoposide- or carboplatin-treated HepG2 cells. (A and B) HepG2 cells were treated with etoposide for $24 \mathrm{~h}$ or carboplatin for $72 \mathrm{~h}$ and the HSP70/gp96 mRNA levels were determined by reverse transcription quantitative polymerase chain reaction. (C and D) Cell culture supernatants were collected and the HSP70/gp96 protein levels were quantified by ELISA. The data shown are representative of four independent experiments for each drug. HSP70, heat shock protein 70 .

malignant and proteins mutate or change, HSPs accumulate to adapt to the condition and protect cells from damage. For example, if tumour cells suffer from stress, such as following
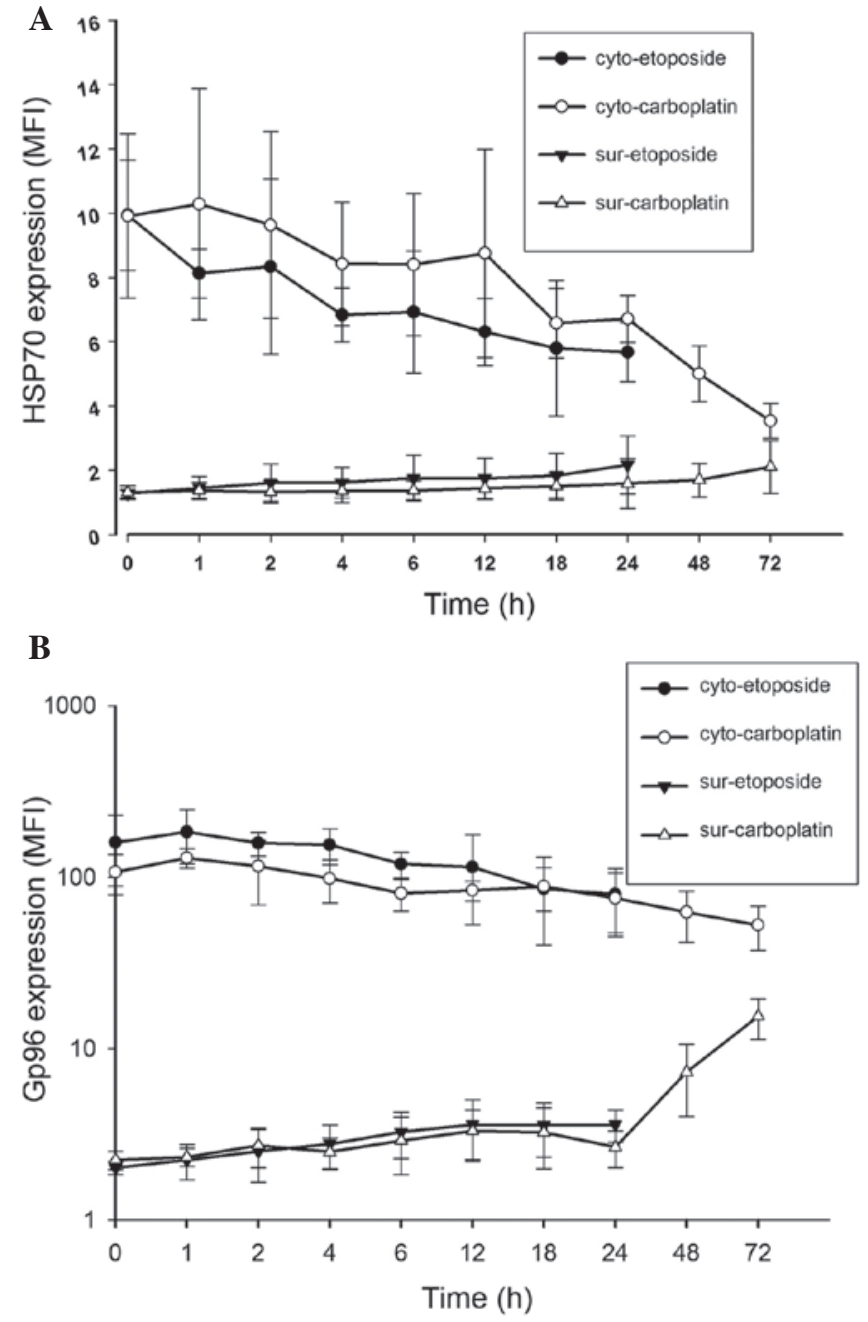

Figure 3. Alterations in cytoplasmic and surface HSP70/gp96 expression in etoposide- or carboplatin-treated HepG2 cells. HepG2 cells were treated with etoposide or carboplatin for the indicated time intervals. (A) Cytoplasmic and surface HSP70/gp96 alterations were determined by flow cytometry. (B) Cytoplasmic and surface gp96 alterations. The data shown are representative of more than four independent experiments. Cyto-etoposide, cytoplasmic HSP70/gp96 expression in etoposide-treated HepG2 cells; cyto-carboplatin, cytoplasmic HSP70/gp96 expression in carboplatin-treated HepG2 cells; sur-etoposide, surface HSP70/gp96 expression in etoposide-treated HepG2 cells; sur-carboplatin, surface HSP70/gp96 expression in carboplatin-treated HepG2 cells. HSP70, heat shock protein 70; MFI, mean fluorescence intensity.

photodynamic therapy (17), HSPs not only markedly increase but also alter their distribution. They translocate from the cytosol to the cell surface and even release into the extracellular environment (23). In this circumstance, antigens chaperoned by HSPs are exposed to immune surveillance elements. Hence, HSP surface expression levels have been reported to correlate with tumour immunogenicity (19) and HSPs are considered to be 'risk signals', alerting to the existence of a threat and priming a self-protection system $(24,25)$. Thus, upregulating cell surface HSPs in cancer cells or stimulating HSP release and exposure may be a crucial method to enhance anti-tumour activity.

Chemotherapy is considered to be an important treatment for HCC, but its acute and cumulative toxicity limit its application. However, immunotherapy, with its different functional mechanism, can be combined with chemotherapy. Chemotherapy is 

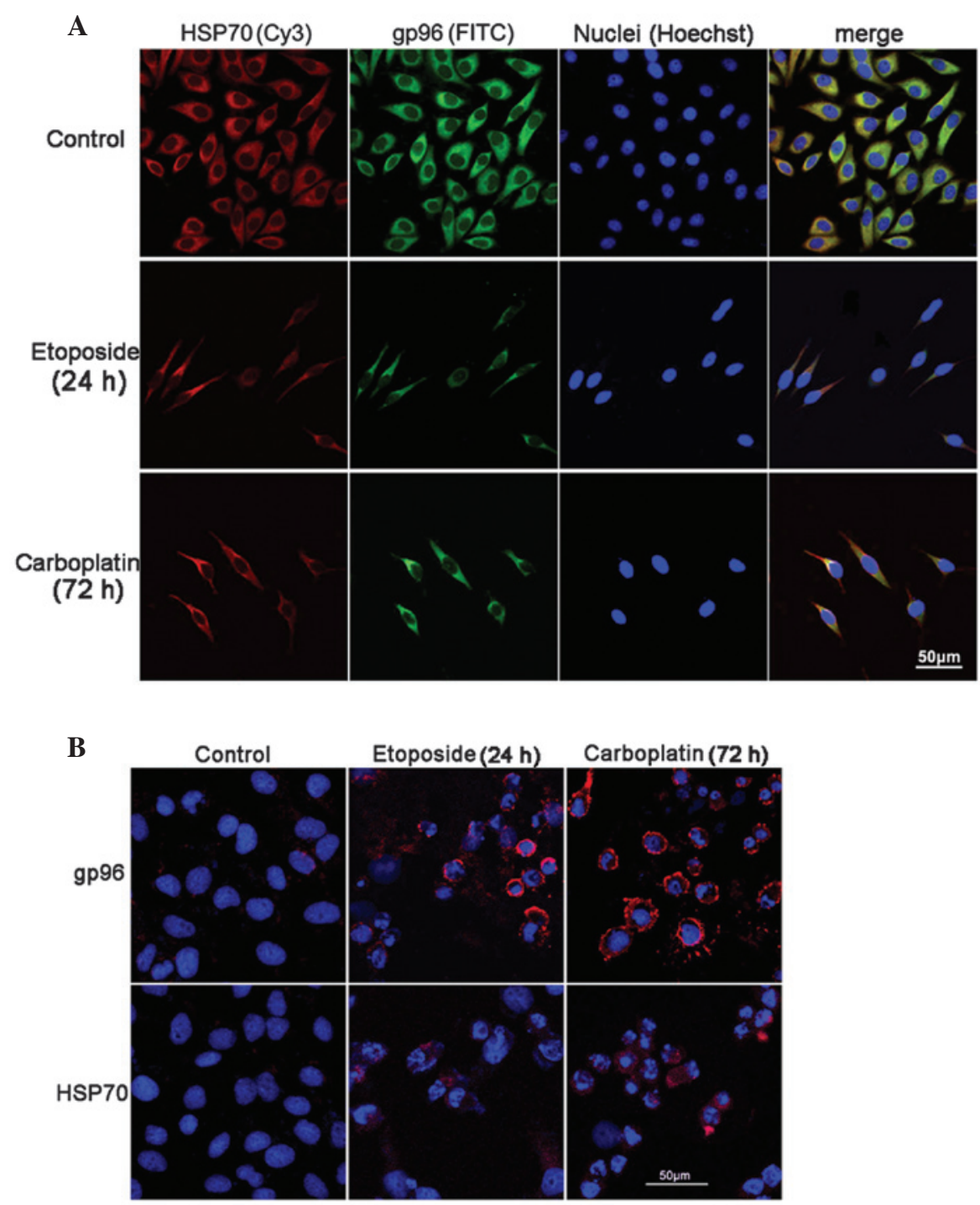

Figure 4. Representative images of cytoplasmic and surface HSP70/gp96 expression in etoposide- or carboplatin-treated HepG2 cells. HepG2 cells were treated with etoposide for $24 \mathrm{~h}$ or carboplatin for $72 \mathrm{~h}$. HSP70/gp96 expression was determined using immunofluorescence staining and confocal images were captured. (A) Cytoplasmic HSP70/gp96 expression. (B) Surface gp96 expression. The images are shown at x400 magnification. FITC, fluorescein isothiocyanate; HSP70, heat shock protein 70 .

an immunological priming factor. It reduces tumour burden, augments tumour immunogenicity and provides the basis for an effective immunoresponse. A rational combination of chemoand immunotherapy would lead to solid tumour regression and generate immunological memory (26). Furthermore, immunotherapy based on HSPs has demonstrated such effectiveness that it is not necessary to identify each tumour-specific antigen (27). In contrast to previous studies in which heat shock, ultraviolet radiation or pathogenic microorganisms were applied as a stressor, the present study employed chemodrugs as a stressor to examine their effects on HSPs in the hepatoma cell line HepG2. The present study primarily detected alterations in HSP70 and gp96 that are well investigated and can aid in eliciting an anticancer immunity response $(28,29)$. As the data show, these chemodrugs were able to cause HepG2 cell apoptosis. The hypersensitive anticancer drug etoposide induced rapid apoptosis of cancer cells and after a $24 \mathrm{~h}$ treatment, the majority of cells died, thus cancer cell apoptosis and HSP alterations could only be recorded for $24 \mathrm{~h}$. Conversely, the hyposensitive anticancer drug carboplatin induced apoptosis relatively slowly and $72 \mathrm{~h}$ were required to reach the peak of apoptosis. Along with demonstrating apoptosis, the cancer cells presented the 'danger signals' HSP70/gp96. Following treatment with etoposide for $24 \mathrm{~h}$ or carboplatin for $72 \mathrm{~h}$, cancer cells were collected to determine the expression of HSP70/gp96. RT-qPCR data demonstrated that the mRNA levels of HSP70/gp96 increased. In addition, when treated with carboplatin for $72 \mathrm{~h}$, HSP70 expression and, even more markedly, gp96 expression exhibited a transfer pattern from the cytoplasm to the cell surface and released into the extracellular environment. This external exposure of HSP70/gp96 may result in an enhancement of tumour immunogenicity. These results have greater significance than the simple upregulation of HSP genes established in previous studies (20). Therefore, it would be beneficial to re-evaluate the effects of hyposensitive drugs and adjust therpeutic stratagies.

Previous studies have verified that HSP-peptide complex could present tumour antigens to activate a specific CTL response (30-32). These studies claimed that HSPs could be 
employed for cancer treatment. However, in cancer patients, increased HSPs did not elicit an effective anti-tumour response and the patients often appeared to be immunotolerant (33). In addition to a large tumour burden, the possibility that the tumour antigens are not fully exposed to the immune surveillance system is an important factor to consider. Therefore, in addition to degrading the tumour burden, challenging tumour cells with a stressor and eliciting antigen exposure and presentation are of significance. The present study successfully employed chemodrugs as a stressor. They induced cancer cell apoptosis and augmented surface HSP70/gp96 expression, resulting in HSP70/gp96 release to the microenvironment. Thus, combined with the HSP70/gp96 'danger signal', apoptotic tumour cells treated with chemodrugs would elicit an immune response and subsequently eradicate residual tumour cells. Notably, the present study found that although the hypersensitive drug etoposide could promote rapid cancer cell apoptosis, it induced less HSP70/gp96 exposure. The continuous treatment of cancer cells by the hyposensitive drug carboplatin was more effective, and it induced stronger surface expression and release of gp96, which suggests a potential use of hyposensitive drugs for HSP-based DC vaccine preparation in vitro and chemoimmunotherapy for HCC patients. The present study provided an experimental basis for patient-specific chemoimmunotherapy for HCC. The results demonstrated that chemodrugs boosted surface HSP expression and release, which may facilitate tumour immunogenicity. Further studies are required to investigate the potential of this therapy.

\section{Acknowledgements}

This study was supported by grants from the National Natural Science Foundation of China (grant no. 81202076) and the Natural Science Foundation of Guangdong to Mei Yang (grant no. S2013010012048) to MY.

\section{References}

1. Tang ZY, Yu YQ, Zhou XD, Ma ZC and Wu ZQ: Progress and prospects in hepatocellular carcinoma surgery. Ann Chir 52: 558-563, 1998

2. Zhou XD, Tang ZY, Yu YQ, et al: Recurrence after resection of alpha-fetoprotein-positive hepatocellular carcinoma. J Cancer Res Clin Oncol 120: 369-373, 1994.

3. Bismuth H, Chiche L, Adam R, Castaing D, Diamond T and Dennison A: Liver resection versus transplantation for hepatocellular carcinoma in cirrhotic patients. Ann Surg 218: 145-151, 1993.

4. Jaeck D, Bachellier P, Oussoultzoglou E, Weber JC and Wolf P: Surgical resection of hepatocellular carcinoma. Post-operative outcome and long-term results in Europe: an overview. Liver Transpl 10 (2 Suppl 1): S58-S63, 2004.

5. El-Serag HB, Marrero JA, Rudolph L and Reddy KR: Diagnosis and treatment of hepatocellular carcinoma. Gastroenterology 134 1752-1763, 2008.

6. Kabilova TO, Kovtonyuk LV, Zonov EV, et al: Immunotherapy of hepatocellular carcinoma with small double-stranded RNA. BMC Cancer 14: 338-349, 2014.

7. Breous $E$ and Thimme R: Potential of immunotherapy for hepatocellular carcinoma. J Hepatol 54: 830-834, 2011.

8. Srivastava P: Roles of heat-shock proteins in innate and adaptive immunity. Nat Rev Immunol 2: 185-194, 2002.

9. Ravoet C, Bleiberg H and Gerard B: Non-surgical treatment of hepatocarcinoma. J Surg Oncol Suppl 3: 104-111, 1993.
10. Zinkernagel RM: Immunity against solid tumors? Int J Cancer 93: $1-5,2001$.

11. Correale P, Cusi MG, Tsang KY, et al: Chemo-immunotherapy of metastatic colorectal carcinoma with gemcitabine plus FOLFOX 4 followed by subcutaneous granulocyte macrophage colony-stimulating factor and interleukin-2 induces strong immunologic and antitumor activity in metastatic colon cancer patients. J Clin Oncol 23: 8950-8958, 2005.

12. Correale P, Cusi MG, Del Vecchio MT, et al: Dendritic cell-mediated cross-presentation of antigens derived from colon carcinoma cells exposed to a highly cytotoxic multidrug regimen with gemcitabine, oxaliplatin, 5-fluorouracil and leucovorin, elicits a powerful human antigen-specific CTL response with antitumor activity in vitro. J Immunol 175: 820-828, 2005.

13. Skoberne M, Beignon AS, Larsson M, et al: Apoptotic cells at the crossroads of tolerance and immunity. Curr Top Microbiol Immunol 289: 259-292, 2005.

14. Kleinclauss F, Perruche S, Masson E, et al: Intravenous apoptotic spleen cell infusion induces a TGF-beta-dependent regulatory T-cell expansion. Cell Death Differ 13: 41-52, 2006.

15. Zheng L, He M, Long M, Blomgran R and Stendahl O: Pathogen-induced apoptotic neutrophils express heat shock proteins and elicit activation of human macrophages. J Immunol 173: 6319-6326, 2004.

16. Feng H, Zeng Y, Whitesell L and Katsanis E: Stressed apoptotic tumor cells express heat shock proteins and elicit tumor-specific immunity. Blood 97: 3505-3512, 2001

17. Korbelik M, Sun J and Cecic I: Photodynamic therapy-induced cell surface expression and release of heat shock proteins: relevance for tumor response. Cancer Res 65: 1018-1026, 2005.

18. Liu B, DeFilippo AM and Li Z: Overcoming immune tolerance to cancer by heat shock protein vaccines. Mol Cancer Ther 1: 1147-1151, 2002.

19. Clark PR and Ménoret A: The inducible Hsp70 as a marker of tumor immunogenicity. Cell Stress Chaperones 6: 121-125, 2001.

20. Li CL, Meng X, Lang ZW and Zhang LJ: The detection of heat shock protein gp96 in primary hepatocellular carcinoma. Zhonghua Gan Zang Bing Za Zhi 12: 569-570, 2004 (In Chinese).

21. Udono H and Srivastava PK: Comparison of tumor-specific immunogenicities of stress-induced proteins gp96, hsp90, and hsp70. J Immunol 152: 5398-5403, 1994.

22. Lancaster GI and Febbraio MA: Exosome-dependent trafficking of HSP70: A novel secretory pathway for cellular stress proteins. J Biol Chem 280: 23349-23355, 2005.

23. Brusa D, Migliore E, Garetto S, Simone M and Matera L: Immunogenicity of 56 degrees $\mathrm{C}$ and UVC-treated prostate cancer is associated with release of HSP70 and HMGB1 from necrotic cells. Prostate 69: 1343-1352, 2009.

24. Beg AA: Endogenous ligands of Toll-like receptors: implications for regulating inflammatory and immune responses. Trends Immunol 23: 509-512, 2002.

25. Matzinger P: The danger model: a renewed sense of self. Science 296: 301-305, 2002.

26. Nowak AK, Robinson BW and Lake RA: Synergy between chemotherapy and immunotherapy in the treatment of established murine solid tumors. Cancer Res 63: 4490-4496, 2003.

27. Ménoret A, Chaillot D, Callahan M and Jacquin C: Hsp70, an immunological actor playing with the intracellular self under oxidative stress. Int J Hyperthermia 18: 490-505, 2002.

28. Singh-Jasuja H, Scherer HU, Hilf N, et al: The heat shock protein gp96 induces maturation of dendritic cells and down-regulation of its receptor. Eur J Immunol 30: 2211-2215, 2000.

29. Millar DG, Garza KM, Odermatt B, et al: Hsp70 promotes antigen-presenting cell function and converts T-cell tolerance to autoimmunity in vivo. Nat Med 9: 1469-1476, 2003.

30. Tamura Y, Peng P, Liu K, Daou M and Srivastava PK: Immunotherapy of tumors with autologous tumor-derived heat shock protein preparations. Science 278: 117-120, 1997.

31. Schild H, Arnold-Schild D, Lammert E and Rammensee HG: Stress proteins and immunity mediated by cytotoxic T lymphocytes. Curr Opin Immunol 11: 109-113, 1999.

32. Sato K, Torimoto Y, Tamura Y, et al: Immunotherapy using heat-shock protein preparations of leukemia cells after syngeneic bone marrow transplantation in mice. Blood 98: 1852-1857, 2001.

33. Pawel S and Anne MD: The immunosuppressive activity of heat shock protein 70. Autoimmune Diseases 2012: 1-6, 2012. 